\title{
Transthoracic Assessment of Coronary Flow Velocity Reserve: A Practical Approach to Diagnostic Testing in Patients with Angina and No Obstructive Coronary Artery Disease
}

\author{
Daria Frestad Bechsgaard (iD) ${ }^{1}$ and Eva Prescott ${ }^{2}$ \\ ${ }^{1}$ Department of Cardiology, North Zealand University Hospital, University of Copenhagen, Dyrehavevej 29, \\ Hillerød 3400, Denmark \\ ${ }^{2}$ Department of Cardiology, Bispebjerg University Hospital, University of Copenhagen, Bispebjerg Bakke 23, \\ Copenhagen 2400, Denmark \\ Correspondence should be addressed to Daria Frestad Bechsgaard; daria.frestad@gmail.com
}

Received 5 October 2020; Revised 16 February 2021; Accepted 18 March 2021; Published 29 March 2021

Academic Editor: Todd J. Anderson

Copyright (C) 2021 Daria Frestad Bechsgaard and Eva Prescott. This is an open access article distributed under the Creative Commons Attribution License, which permits unrestricted use, distribution, and reproduction in any medium, provided the original work is properly cited.

\begin{abstract}
More than half of the patients with symptoms suggestive of myocardial ischemia presenting at invasive angiography have no obstructive coronary artery disease (CAD). A large proportion of these patients have ischemia caused by coronary microvascular dysfunction, a condition associated with adverse cardiovascular prognosis. Measurement of coronary flow velocity reserve by transthoracic Doppler echocardiography is a feasible and reproducible method for the evaluation of coronary microvascular function. This review provides a practical overview of the method in a clinical setting of angina and noobstructive CAD, including technical details and prognostic significance.
\end{abstract}

\section{Introduction}

The discrepancy between angina symptoms, positive stress tests, and no evidence of flow-limiting stenosis on invasive angiography is a common diagnostic challenge, more prevalent in women than in men [1-3]. No evidence of obstructive coronary artery disease (CAD) often results in no diagnosis and limited treatment options, yet follow-up has revealed an increased risk of cardiovascular events in these patients compared with an age- and sex-matched reference population $[2,4]$. Over the last two decades, abnormalities of cardiac microvascular function have received increased attention, including coronary microvascular dysfunction (CMD), as the possible explanation for the continued symptoms and adverse cardiovascular prognosis in these patients $[5,6]$. CMD is a dysfunction of the coronary resistance vessels, causing a mismatch between coronary blood supply and myocardial oxygen demand. Transthoracic Doppler echocardiography (TTDE) is an established method of assessment of coronary microvascular function with a well-documented prognostic significance [7]. The main focus of this review is to provide a practical overview of the TTDE-guided evaluation of coronary microvascular function, including technical details and common pitfalls.

\section{The Concept of Coronary Flow Velocity Reserve}

The coronary arterial system comprises a network of vessels with distinct functional properties. Epicardial arteries are conductance vessels with capacitance function offering little resistance to coronary blood flow. Prearterioles and arterioles are resistance vessels sensitive to changes in shear stress and perfusion pressure, responsible for the regulation and distribution of coronary blood flow. Their main function is to prevent myocardial ischemia by matching the oxygen supply with the dynamics of myocardial oxygen demand. The energy production in the normal heart primarily depends on oxidative phosphorylation; thus, an increase in cardiac activity demands for an adequate increase in oxygen 
supply. Because the myocardium already extracts more than $70 \%$ of the oxygen delivered, this can only be met by an increase in coronary blood flow. Under physiological conditions, the mechanism of autoregulation allows for up to a 5 -fold increase in coronary blood flow to meet the oxygen demand with increased cardiac activity [8].

Coronary microvascular vessels are beyond the resolution of the current angiographic systems; however, their function can be assessed indirectly, e.g., through measurements of steady-state coronary blood flow velocities during rest and pharmacologically induced hyperemia using TTDE. In the absence of significant obstructive $\mathrm{CAD}$, the ratio of hyperemic to resting coronary flow velocities $(\mathrm{m} / \mathrm{s})$, coronary flow velocity reserve (CFVR), is an established physiological estimate of coronary microvascular function (Figure 1) [9]. The measured increase in coronary flow velocity equals the increase in total myocardial flow if epicardial vessel diameter is constant. TTDE CFVR is measured on a continuous scale, and cutoffs of 2 or 2.5 have been used and are associated with adverse cardiovascular outcomes in angina patients with no obstructive CAD [10].

\section{Technical Considerations}

TTDE assessment of coronary microvascular function is summarized in Table 1. Coronary flow velocities can be measured in all three major coronary arteries, and the choice of the vessel is often determined by feasibility. There are no large-scale studies comparing CFVR in all three vessels in unselected patient cohorts; however, the existing literature suggests the highest CFVR feasibility rates (up to 100\%) in the left anterior descending artery (LAD), followed by the right and circumflex coronary arteries $[11,12]$. The following review is, therefore, focused on the LAD-CFVR evaluation.

Proximal to distal segments of LAD can be visualized by a 2D color Doppler in a modified parasternal short-axis view, parasternal long-axis view, and foreshortened apical four- or two-chamber views, using a $2.7-8 \mathrm{MHz}$ transducer, with the patient laid in a stable left lateral decubitus position [13]. The color Doppler velocity range is set between 10 and $25 \mathrm{~cm} / \mathrm{s}$, and the baseline color scale is set between 1.00 and $2.50 \mathrm{kHz}$, depending on low or high flow velocities, respectively $[11,14,15]$.

Several factors may affect the quality and validity of a CFVR assessment. Use of adenosine, dipyridamole, or regadenoson as a stressor requires 24 -hour abstinence from drinks and food containing significant amount of methylxanthines (e.g., coffee, tea, soda, energy drinks, chocolate, and banana) which block adenosine receptors. [16] Medications containing dipyridamole should be paused for at least 48 hours, and medications affecting myocardial perfusion or myocardial metabolic activity (e.g., nitrates, $\beta$-blockers, and antihypertensives) should be paused for at least 24 hours. Thorough patient preparations, including breathing exercises and comfortable positioning, are of essence to prevent probe displacement due to body movements or increased breathing activity during hyperemia.

Identification, alignment, and fixation of the vessel are the most important and time-consuming steps in the process
(Figure 2). The artery is identified using Doppler flow mapping. Color gain can be adjusted to obtain the optimal image quality. Moreover, if visualization is challenging, intravenous contrast enhancement in refracted doses can be used. Coronary flow velocity is measured by using a pulsedwave Doppler as a laminar flow signal directed toward the transducer. The ultrasound beam should be aligned as parallel to the coronary flow as technically possible. Angle correction for coronary flow velocity measurements is not routinely applied due to CFVR being a ratio; however, it is essential that the ultrasound beam is kept under the same angle during both rest and hyperemia to avoid measurement errors. The pattern of coronary flow is biphasic with the highest flow during diastole. Both 2D color Doppler and pulsed-wave Doppler images should be stored frequently throughout the examination to document probe positioning and sampling angle and to capture the peak flow velocities during rest and hyperemia. In addition, heart rate and blood pressure should be documented frequently during rest, hyperemia, and once after discontinuation of the vasodilator.

3.1. Vasodilators. The most common vasodilators used for TTDE CFVR evaluation are adenosine, dipyridamole, and regadenoson. Adenosine $(0.14 \mathrm{mg} / \mathrm{kg} / \mathrm{minute}$; infusion) induces microvascular dilatation through activation of A2A receptors [17]. This results in a 3- to 4-fold increase in coronary blood flow in a normal epicardial vessel. Due to the short half-life of adenosine ( $<10$ seconds), there is no need for an antidote and most side effects resolve in a few seconds after discontinuation of the adenosine infusion. The most common side effects are flushing, chest tightness, and shortness of breath. Less common but more serious side effects are AV block and bronchospasm [18]. In some patients, shortness of breath during adenosine infusion can be dominating, leading to an increased chest movement and higher risk of probe dispositioning and measure error. Dipyridamole $(0.84 \mathrm{mg} / \mathrm{kg}$; infusion) inhibits reuptake of endogenic adenosine and has a similar effect on coronary microcirculation. The side effect profile is, however, slightly different. The most common side effects are dizziness, chest and abdominal discomfort, and headache [19]. Dipyridamole has a significantly longer half-life, and administration of an antidote (aminophylline; $50-250 \mathrm{mg}$ ) is often necessary. Like adenosine, the selective A2A receptor agonist regadenoson ( $0.4 \mathrm{mg}$; rapid injection) dilates the coronary microvasculature by acting on the smooth muscle cells. The most common side effects are shortness of breath, headache, and flushing, which resolve within 15 minutes of administration. Persisting adverse reactions can be attenuated using aminophylline. These three vasodilators assess the nonendothelial dependent pathway of coronary microvascular function in the absence of epicardial coronary stenosis, although the effect is also to some part mediated by endothelial release of nitric oxide [20]. Furthermore, adenosine and dipyridamole have previously been considered equal to achieve hyperemia and are used interchangeably in TTDE [21]. 


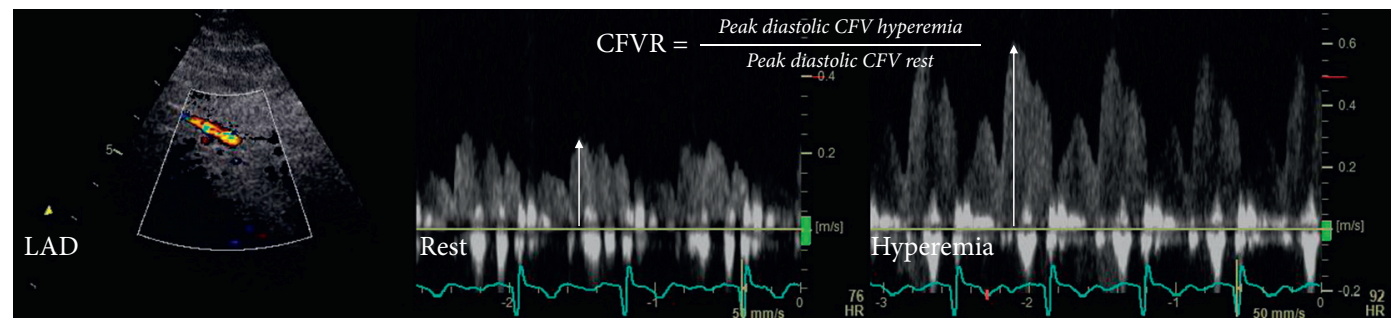

FIGURE 1: Coronary flow velocity reserve (CFVR) measured in the left anterior descending artery (LAD).

TABle 1: Noninvasive assessment of coronary microvascular function by transthoracic Doppler echocardiography.

Summary of noninvasive assessment of coronary microvascular function by transthoracic Doppler echocardiography

Physiological pathway: nonendothelial dependent

Preferred coronary artery: left anterior descending artery

Measurement: coronary flow velocity reserve (CFVR) ratio of hyperemic to resting coronary flow velocities (m/s); continuous scale

Cutoff for coronary microvascular dysfunction: CFR $<2.0$

Common vasodilators: adenosine $(0.14 \mathrm{mg} / \mathrm{kg} / \mathrm{minute}$; intravenous infusion); dipyridamole $(0.84 \mathrm{mg} / \mathrm{kg}$; intravenous infusion); and regadenoson ( $0.4 \mathrm{mg}$; intravenous injection)

Patient preparation: absence from methylxanthines and medications affecting myocardial perfusion or myocardial metabolic activity; breathing exercises

Examination steps:

(i) Identification of the coronary flow signal using a $2 \mathrm{D}$ color Doppler or intravenous contrast enhancement in case of poor visualization

(ii) Alignment of the coronary flow signal of the ultrasound beam as parallel to the coronary flow as possible

(iii) Maintenance of probe position and measuring angle throughout the examination

(iv) Documentation of characteristic flow curves during rest and hyperemia

(v) CFVR quality considerations [11]

Common pitfalls:

(i) Loss of coronary flow signal/change in measuring angle due to patient/probe displacement

(ii) Alternating peak flow velocities due to coronary tortuosity/multiple vessels

(iii) Noise artefacts mimicking/blurring coronary flow signal

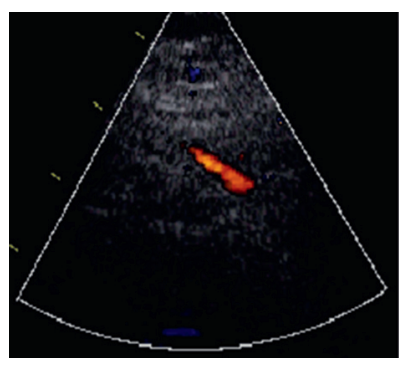

(a)

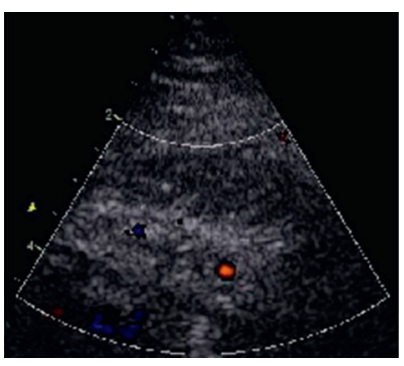

(b)

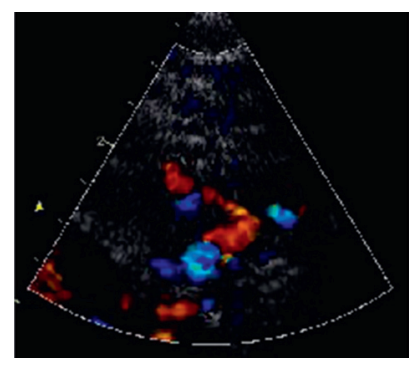

(c)

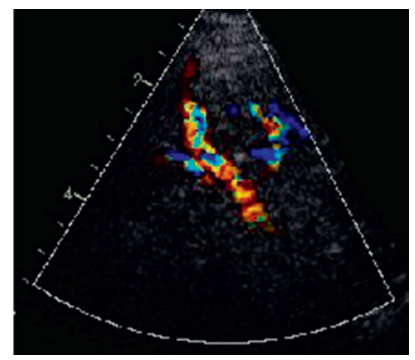

(d)

Figure 2: 2D color Doppler view of the left anterior descending artery (LAD) demonstrating the importance of artery identification and alignment for accurate CFVR estimation. (a) Optimal visualization of the LAD segment in a foreshortened two-chamber view. Suboptimal artery identification compromising quality of CFVR evaluation: (b) only a short segment of the artery visible; (c) tortuous artery; and (d) multiple vessels in the same frame.

Simultaneous assessment of left ventricular regional wall motion can be performed during dipyridamole and adenosine infusions [22].

3.2. Feasibility and Variability. Published reports show that TTDE CFVR in the LAD is highly reproducible in experienced hands. In a large study of angina patients with no obstructive CAD ( $n=947)$, CFVR of the LAD was feasible in $97 \%$ of patients, with only $6 \%$ of all examinations performed using an intravenous contrast agent [11]. Smaller studies have reported feasibility rates between $66 \%$ and $100 \%$ [23-27]. The feasibility is lower in the circumflex and right coronary arteries. Common factors affecting feasibility are operator experience and patient-related factors (high BMI, diabetes, and presence of nonobstructive atherosclerosis) [11]. Good repeatability (repeated examinations) of CFVR in the LAD has previously been reported for both healthy volunteers and various patient populations [6, 23, 28-31]. Low intra- and interreader variations have been reported by 
several research groups, suggesting good reproducibility (repeated readings) of CFVR $[6,28,32]$. The good repeatability and reproducibility of TTDE CFVR supports the use of CFVR in serial evaluations in both clinical and research settings, as well as an outcome measure.

TTDE CFVR in the LAD is closely correlated with CFVR measured using an intracoronary Doppler guidewire in patients undergoing angiography for suspected obstructive CAD [23, 25-27]. A few studies have explored the agreement between TTDE CFVR and the current noninvasive gold standard for the evaluation of coronary microvascular function, positron emission tomography, in various patient populations, reporting a wide range of correlation coefficients (ranging between 0.27 and 0.91 ), and there is no clear agreement between the methods [28, 30, 31]. However, also test-retest properties of positron emission tomography are suboptimal with a $\mathrm{CoV}$ of approximately $20 \%$ in healthy individuals [33]. Other noninvasive methods include cardiac magnetic resonance imaging and CT-perfusion. These have, however, not yet been standardized or validated for use in diagnosing coronary microvascular function. Small studies have found nonsignificant correlations between coronary microvascular function assessed using TTDE and cardiac magnetic resonance perfusion imaging, suggesting perhaps that techniques measuring coronary flow and myocardial perfusion are not interchangeable in the evaluation of coronary microvascular function $[34,35]$.

\subsection{Factors Affecting the Quality of Coronary Flow Velocity} Measurement. Several patient- and non-patient-related factors may influence the quality and validity of CFVR measurements (Figures 2-4). To date, there is no consensus on the quality score for TTDE CFVR. The iPOWER (Improve Diagnosis and Treatment of Women with Angina Pectoris and Microvessel Disease) research group has suggested a semiquantitative quality score, based on a large, unselected sample of women $(n=947)$ with angina and no obstructive disease [11]. The score (0 [nonfeasible], 1 [low quality], 2 [medium quality], and 3 [high quality]) was based on 4 main criteria, including (1) vessel identification, (2) maintenance of probe position throughout the examination, (3) visibility and configuration of coronary flow in the $2 \mathrm{D}$ color Doppler mode, and (4) characteristics of flow curves in the pulsed-wave mode [11]. Identification of a single vessel without confounding side branches, good visibility and parallel alignment of beam direction to the vessel flow, consistent probe positioning throughout the entire examination, and characteristic biphasic flow curves gradually increasing during hyperemia with well-defined peaks would classify as a high-quality examination.

The most common pitfall during CFVR assessment is the loss of coronary flow signal or significant shift in measurement angle due to patient or probe displacement (e.g., uncomfortable positioning) or increased chest movements due to side effects of the stress agent, underscoring the importance of proper patient preparation. Another pitfall is alternating peak flow velocities due to arterial tortuosity or multiple vessels measured by the pulsed wave (Figure 2).
Furthermore, noise from the pericardial space (fluid or fat) can sometimes mimic coronary flow, producing uncharacteristic flow curves. These errors lead to nonfeasible examinations and, if not recognized, to under- or overestimation of CFVR.

3.4. Factors Associated with CFVR. According to the current European guidelines, CFVR $<2$ indicates impaired coronary microvascular function [36]. The current cutoff is based on studies investigating a broad spectrum of patients with various risk factor profiles and stages of $\mathrm{CAD}$, ranging from normal epicardial arteries to obstructive CAD [15]. CFVR has previously been associated with several risk factors, including age, hypertension, diabetes, smoking status, resting heart rate, and dyslipidemia [37-43]. However, recent studies investigating women with angina and no obstructive CAD have come to a conclusion that conventional cardiovascular risk factors account for little of the variation in CFVR in these patients [37, 39, 44]. A CFVR cutoff of 2 has also been associated with significant $\mathrm{CAD}$ and regional myocardial ischemia [45-48]. A few studies have reported the usefulness of TTDE CFVR to assess the functional significance of intermediate coronary artery stenosis $[48,49]$. Currently, there is a knowledge gap on the association between CFVR and plaque burden in patients with nonobstructive CAD.

\section{Prognostic Value of CFVR}

Increasing amount of literature suggests that CMD evaluated by TTDE CFVR predicts adverse cardiovascular outcomes in patients without obstructive CAD. In a meta-analysis performed by our group, including 4 prognostic studies evaluating patients with stable angina and no obstructive CAD (4.516 patients; 284 events), the pooled relative risk for cardiovascular events (incident fatal and nonfatal coronary heart disease) was 4.57 (95\% CI 3.43-6.08) [7, 50-53]. A similar prognostic value of CFVR assessed by PET has been reported [7, 54].

\section{Clinical Application of TTDE CFVR}

Angina patients without obstructive CAD are often underdiagnosed and undertreated and at higher risk of hospital readmissions, repeated invasive diagnostic procedures, depression and vital exhaustion, reduced quality of life, and premature exit from the workforce [55-57]. Evaluation of coronary microvascular function using TTDE CFVR in these patients is recommended by ESC guidelines (Class IIb recommendation); however, despite TTDE being a noninvasive, low-cost, and radiation-free method, it is not routinely implemented in clinical practice due to its limited availability [36]. TTDE is an operator-dependent imaging modality; thus, the quality of CFVR largely depends on operator skills and experience. TTDE CFVR performed by an experienced operator is feasible, reproducible, and correlates well with the invasive gold standard. Increasing knowledge and awareness of the adverse prognosis associated with myocardial ischemia in the absence of flow-limiting CAD warrants a wider use of noninvasive diagnostic 


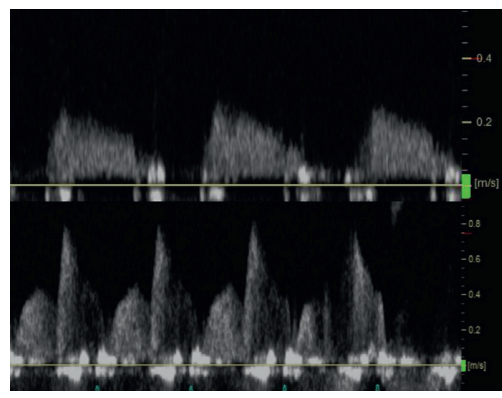

(a)

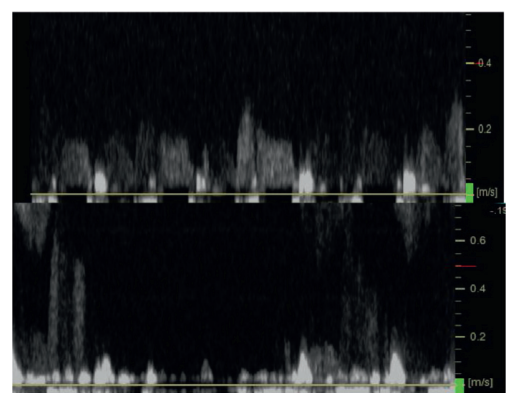

(b)

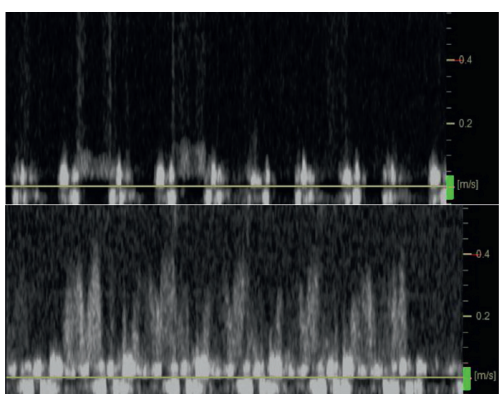

(c)

Figure 3: Examples of different qualities of coronary flow velocity curves at rest (upper level) and peak hyperemia (lower level). (a) Welldefined and reproducible flow curves. (b) Blurred and inconsistent flow curves (e.g., due to chest movements). (c) Poorly defined flow curves (e.g., due to probe displacement leading to partial loss of coronary flow signal).

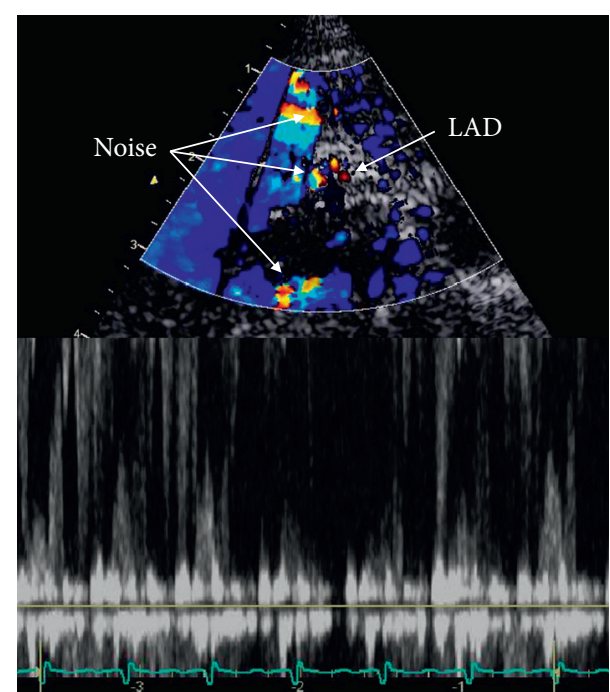

FIGURE 4: Example of noise artifact compromising coronary flow signal, making coronary flow velocity curves blurred and poorly reproducible. Noise artifacts can occur from surrounding structures (e.g., vessels, epicardial fat, and flow in the left ventricular cavity) and can sometimes mimic coronary flow as they usually increase during hyperemia.

techniques, including TTDE, in clinical evaluation of coronary microvascular function.

Evaluation of coronary microvascular function can benefit angina patients in terms of a diagnosis and symptom management. Currently, there is no evidence-based treatment of CMD; however, management of lifestyle factors and risk factors may have a beneficial effect [55]. According to the recent CorMica (Coronary Microvascular Angina) trial, a patient-centered approach, including evaluation of coronary microcirculation as an add-on procedure to diagnostic invasive angiography, linked together with medical therapy (antianginal and prevention therapies, including lifestyle modification), is feasible and improves angina in patients with no obstructive CAD [58]. Looking beyond angina patients with nonobstructive $\mathrm{CAD}, \mathrm{CMD}$ can be present in other clinical settings, including myocardial diseases (e.g., hypertrophic or dilated cardiomyopathy and amyloidosis), aortic stenosis, and obstructive CAD [59]. However, evidence on the effect of therapy on CMD associated with these conditions is largely lacking.

\section{Conclusions}

The noninvasive assessment of coronary microvascular function by TTDE CFVR in the LAD is an established method with documented prognostic significance. Compared with other noninvasive methods (e.g., cardiac magnetic resonance imaging or positron emission tomography), TTDE CFVR is an inexpensive, readily available, nonradiative procedure and can be performed simultaneously with diagnostic transthoracic echocardiography as an addon examination. CFVR by TTDE is feasible and reproducible; however, the method is technically challenging and requires extensive operator experience. There is currently no agreement upon the quality score for CFVR. Large, multicenter trials are warranted to establish the value of TTDE CFVR in cardiovascular risk stratification in patients with no obstructive CAD. Furthermore, the role of TTDE CFVR in guiding symptom management and evaluation of prevention and potential treatment therapies is yet to be established.

\section{Data Availability}

No data were used in this study.

\section{Conflicts of Interest}

The authors declare no conflicts of interest.

\section{References}

[1] M. R. Patel, E. D. Peterson, D. Dai et al., "Low diagnostic yield of elective coronary angiography," New England Journal of Medicine, vol. 362, no. 10, pp. 886-895, 2010.

[2] C. N. Bairey Merz, C. J. Pepine, M. N. Walsh, and J. L. Fleg, "Ischemia and No obstructive coronary artery disease (INOCA)," Circulation, vol. 135, no. 11, pp. 1075-1092, 2017.

[3] M. R. Patel, D. Dai, A. F. Hernandez et al., "Prevalence and predictors of nonobstructive coronary artery disease identified with coronary angiography in contemporary clinical practice," American Heart Journal, vol. 167, no. 6, pp. 846-852, 2014. 
[4] L. Jespersen, A. Hvelplund, S. Z. Abildstrom et al., "Stable angina pectoris with no obstructive coronary artery disease is associated with increased risks of major adverse cardiovascular events," European Heart Journal, vol. 33, no. 6, pp. 734-744, 2012.

[5] C. N. Merz, S. F. Kelsey, C. J. Pepine et al., "The women's ischemia syndrome evaluation (WISE) study: protocol design, methodology and feasibility report," Journal of the American College of Cardiology, vol. 33, no. 6, pp. 1453-1461, 1999.

[6] E. Prescott, S. Z. Abildstrøm, A. Aziz et al., "Improving diagnosis and treatment of women with angina pectoris and microvascular disease: the iPOWER study design and rationale," American Heart Journal, vol. 167, no. 4, pp. 452-458, 2014.

[7] P. Brainin, D. Frestad, and E. Prescott, "The prognostic value of coronary endothelial and microvascular dysfunction in subjects with normal or non-obstructive coronary artery disease: a systematic review and meta-analysis," International Journal of Cardiology, vol. 254, pp. 1-9, 2018.

[8] F. Crea, G. A. Lanza, and P. G. Camici, Coronary Microvascular Dysfunction, Springer, New York, NY, USA, 2014.

[9] K. L. Gould and K. Lipscomb, "Effects of coronary stenoses on coronary flow reserve and resistance," The American Journal of Cardiology, vol. 34, no. 1, pp. 48-55, 1974.

[10] A. I. Löffler and J. M. Bourque, "Coronary microvascular dysfunction, microvascular angina, and management," Current Cardiology Reports, vol. 18, pp. 1-7, 2016.

[11] M. M. Michelsen, A. Pena, N. D. Mygind et al., "Coronary flow velocity reserve assessed by transthoracic doppler: the iPOWER study: factors influencing feasibility and quality," Journal of the American Society of Echocardiography, vol. 29, no. 7, pp. 709-716, 2016.

[12] J. Vegsundvåg, E. Holte, R. Wiseth, K. Hegbom, and T. Hole, "Coronary flow velocity reserve in the three main coronary arteries assessed with transthoracic Doppler: a comparative study with quantitative coronary angiography," Journal of the American Society of Echocardiography, vol. 24, no. 7, pp. 758-767, 2011.

[13] M. Krzanowski, W. Bodzoń, and P. P. Dimitrow, "Imaging of all three coronary arteries by transthoracic echocardiography. An illustrated guide," Cardiovascular Ultrasound, vol. 1, no. 1, p. 16, 2003.

[14] T. Wada, K. Hirata, Y. Shiono et al., "Coronary flow velocity reserve in three major coronary arteries by transthoracic echocardiography for the functional assessment of coronary artery disease: a comparison with fractional flow reserve," European Heart Journal-Cardiovascular Imaging, vol. 15, no. 4, pp. 399-408, 2014.

[15] P. Meimoun and C. Tribouilloy, "Non-invasive assessment of coronary flow and coronary flow reserve by transthoracic doppler echocardiography: a magic tool for the real world," European Journal of Echocardiography, vol. 9, no. 4, pp. 449-457, 2008.

[16] C. E. Müller and K. A. Jacobson, "Xanthines as adenosine receptor antagonists," Handbook of Experimental Pharmacology, vol. 200, pp. 151-199, 2011.

[17] T. W. Hein, W. Wang, B. Zoghi, M. Muthuchamy, and L. Kuo, "Functional and molecular characterization of receptor subtypes mediating coronary microvascular dilation to adenosine," Journal of Molecular and Cellular Cardiology, vol. 33, no. 2, pp. 271-282, 2001.

[18] R. Saab and F. G. Hage, "Vasodilator stress agents for myocardial perfusion imaging," Journal of Nuclear Cardiology, vol. 24, no. 2, pp. 434-438, 2017.
[19] S.-D. Lee, W.-C. Huang, N.-J. Peng, and C. Hu, "Dipyridamole-induced adverse effects in myocardial perfusion scans: dynamic evaluation," IJC Heart \& Vasculature, vol. 14, pp. 14-19, 2016.

[20] P. Smits, S. B. Williams, D. E. Lipson, P. Banitt, G. A. Rongen, and M. A. Creager, "Endothelial release of nitric oxide contributes to the vasodilator effect of adenosine in humans," Circulation, vol. 92, no. 8, pp. 2135-2141, 1995.

[21] H. E. Lim, W. J. Shim, H. Rhee et al., "Assessment of coronary flow reserve with transthoracic doppler echocardiography: comparison among adenosine, standard-dose dipyridamole, and high-dose dipyridamole," Journal of the American Society of Echocardiography, vol. 13, no. 4, pp. 264-270, 2000.

[22] P. A. Pellikka, A. Arruda-Olson, F. A. Chaudhry et al., "Guidelines for performance, interpretation, and application of stress echocardiography in ischemic heart disease: from the American society of echocardiography," Journal of the American Society of Echocardiography, vol. 33, no. 1, pp. 1-41, 2020.

[23] C. Caiati, C. Montaldo, N. Zedda et al., "Validation of a new noninvasive method (contrast-enhanced transthoracic second harmonic echo doppler) for the evaluation of coronary flow reserve," Journal of the American College of Cardiology, vol. 34, no. 4, pp. 1193-1200, 1999.

[24] S. M. Kim, W. J. Shim, H. E. Lim et al., "Assessment of coronary flow reserve with transthoracic Doppler echocardiography: comparison with intracoronary Doppler method," Journal of Korean Medical Science, vol. 15, no. 2, pp. 139-145, 2000.

[25] D. J. R. Hildick-Smith, R. Maryan, and L. M. Shapiro, "Assessment of coronary flow reserve by adenosine transthoracic echocardiography: validation with intracoronary doppler," Journal of the American Society of Echocardiography, vol. 15, no. 9, pp. 984-990, 2002.

[26] T. Hozumi, K. Yoshida, T. Akasaka et al., "Noninvasive assessment of coronary flow velocity and coronary flow velocity reserve in the left anterior descending coronary artery by doppler echocardiography," Journal of the American College of Cardiology, vol. 32, no. 5, pp. 1251-1259, 1998.

[27] H. Lethen, H. P. Tries, J. Brechtken, S. Kersting, and H. Lambertz, "Comparison of transthoracic doppler echocardiography to intracoronary doppler guidewire measurements for assessment of coronary flow reserve in the left anterior descending artery for detection of restenosis after coronary angioplasty," The American Journal of Cardiology, vol. 91, no. 4, pp. 412-417, 2003.

[28] M. Saraste, J. W. Koskenvuo, J. Knuuti et al., "Coronary flow reserve: measurement with transthoracic Doppler echocardiography is reproducible and comparable with positron emission tomography," Clinical Physiology, vol. 21, no. 1, pp. 114-122, 2001.

[29] M. Galderisi, S. Cicala, A. D’Errico, O. De Divitiis, and G. De Simone, "Nebivolol improves coronary flow reserve in hypertensive patients without coronary heart disease," Journal of Hypertension, vol. 22, no. 11, pp. 2201-2208, 2004.

[30] M. M. Michelsen, N. D. Mygind, A. Pena et al., "Transthoracic doppler echocardiography compared with positron emission tomography for assessment of coronary microvascular dysfunction: the iPOWER study," International Journal of Cardiology, vol. 228, pp. 435-443, 2017.

[31] R. H. Olsen, L. R. Pedersen, M. Snoer et al., "Coronary flow velocity reserve by echocardiography: feasibility, reproducibility and agreement with PET in overweight and obese 
patients with stable and revascularized coronary artery disease," Cardiovascular Ultrasound, vol. 14, no. 1, p. 22, 2016.

[32] M. Snoer, T. Monk-Hansen, R. H. Olsen et al., "Coronary flow reserve as a link between diastolic and systolic function and exercise capacity in heart failure," European Heart JournalCardiovascular Imaging, vol. 14, no. 7, p. 677, 2012.

[33] C. Byrne, A. Kjaer, N. E. Olsen, J. L. Forman, and P. Hasbak, "Test-retest repeatability and software reproducibility of myocardial flow measurements using rest/adenosine stress Rubidium-82 PET/CT with and without motion correction in healthy young volunteers," Journal of Nuclear Cardiology, 2020.

[34] L. E. J. Thomson, J. Wei, M. Agarwal et al., "Cardiac magnetic resonance myocardial perfusion reserve index is reduced in women with coronary microvascular dysfunction," Circulation: Cardiovascular Imaging, vol. 8, no. 4, 2015.

[35] N. D. Mygind, A. Pena, M. Mide Michelsen et al., "Myocardial first pass perfusion assessed by cardiac magnetic resonance and coronary microvascular dysfunction in women with angina and no obstructive coronary artery disease," Scandinavian Journal of Clinical and Laboratory Investigation, vol. 79, no. 4, pp. 238-246, 2019.

[36] J. Knuuti, W. Wijns, A. Saraste et al., "2019 ESC Guidelines for the diagnosis and management of chronic coronary syndromes," European Heart Journal, vol. 41, no. 3, pp. 407-477, 2020 .

[37] N. D. Mygind, M. M. Michelsen, A. Pena et al., "Coronary microvascular function and cardiovascular risk factors in women with angina pectoris and no obstructive coronary artery disease: the iPOWER study," Journal of the American Heart Association, vol. 5, no. 3, 2016.

[38] C. J. Pepine, R. D. Anderson, B. L. Sharaf et al., "Coronary microvascular reactivity to adenosine predicts adverse outcome in women evaluated for suspected ischemia," Journal of the American College of Cardiology, vol. 55, no. 25, pp. 2825-2832, 2010.

[39] J. D. Sara, R. J. Widmer, Y. Matsuzawa, R. J. Lennon, L. O. Lerman, and A. Lerman, "Prevalence of coronary microvascular dysfunction among patients with chest pain and nonobstructive coronary artery disease," JACC: Cardiovascular Interventions, vol. 8, no. 11, pp. 1445-1453, 2015.

[40] R. Sicari, F. Rigo, L. Cortigiani, S. Gherardi, M. Galderisi, and E. Picano, "Additive prognostic value of coronary flow reserve in patients with chest pain syndrome and normal or nearnormal coronary arteries," The American Journal of Cardiology, vol. 103, no. 5, pp. 626-631, 2009.

[41] S. A. L. Ahmari, T. J. Bunch, K. Modesto et al., "Impact of individual and cumulative coronary risk factors on coronary flow reserve assessed by dobutamine stress echocardiography," The American Journal of Cardiology, vol. 101, no. 12, pp. 1694-1699, 2008.

[42] B. Tuccillo, M. Accadia, S. Rumolo et al., "Factors predicting coronary flow reserve impairment in patients evaluated for chest pain: an ultrasound study," Journal of Cardiovascular Medicine, vol. 9, no. 3, pp. 251-255, 2008.

[43] D.-H. Lee, H.-J. Youn, Y.-S. Choi et al., "Coronary flow reserve is a comprehensive indicator of cardiovascular risk factors in subjects with chest pain and normal coronary angiogram," Circulation Journal, vol. 74, no. 7, pp. 1405-1414, 2010.

[44] T. R. Wessel, C. B. Arant, S. P. McGorray et al., "Coronary microvascular reactivity is only partially predicted by atherosclerosis risk factors or coronary artery disease in women evaluated for suspected ischemia: results from the NHLBI women's ischemia syndrome evaluation (WISE)," Clinical Cardiology, vol. 30, no. 2, pp. 69-74, 2007.

[45] M. Daimon, H. Watanabe, H. Yamagishi et al., "Physiologic assessment of coronary artery stenosis without stress tests: noninvasive analysis of phasic flow characteristics by transthoracic doppler echocardiography," Journal of the American Society of Echocardiography, vol. 18, no. 9, pp. 949-955, 2005.

[46] P. Voci, F. Pizzuto, E. Mariano, P. E. Puddu, P. A. Chiavari, and F. Romeo, "Measurement of coronary flow reserve in the anterior and posterior descending coronary arteries by transthoracic doppler ultrasound," The American Journal of Cardiology, vol. 90, no. 9, pp. 988-991, 2002.

[47] Y. Matsumura, T. Hozumi, H. Watanabe et al., "Cut-off value of coronary flow velocity reserve by transthoracic doppler echocardiography for diagnosis of significant left anterior descending artery stenosis in patients with coronary risk factors," The American Journal of Cardiology, vol. 92, no. 12, pp. 1389-1393, 2003.

[48] P. Meimoun, T. Benali, S. Sayah et al., "Evaluation of left anterior descending coronary artery stenosis of intermediate severity using transthoracic coronary flow reserve and dobutamine stress echocardiography," Journal of the American Society of Echocardiography, vol. 18, no. 12, pp. 12331240, 2005.

[49] H. Okayama, T. Sumimoto, G. Hiasa et al., "Assessment of intermediate stenosis in the left anterior descending coronary artery with contrast-enhanced transthoracic doppler echocardiography," Coronary Artery Disease, vol. 14, no. 3, pp. 247-254, 2003.

[50] K. Nakanish, S. Fukuda, K. Shimada et al., "Impaired coronary flow reserve as a marker of microvascular dysfunction to predict long-term cardiovascular outcomes, acute coronary syndrome and the development of heart failure," Circulation Journal, vol. 76, no. 8, pp. 1958-1964, 2012.

[51] E. Balázs, K. S. Pintér, A. Egyed, M. Csanády, T. Forster, and A. Nemes, "The independent long-term prognostic value of coronary flow velocity reserve in female patients with chest pain and negative coronary angiograms (Results from the SZEGED study)," International Journal of Cardiology, vol. 146, no. 2, pp. 259-261, 2011.

[52] J. A. Lowenstein, C. Caniggia, G. Rousse et al., "Coronary flow velocity reserve during pharmacologic stress echocardiography with normal contractility adds important prognostic value in diabetic and nondiabetic patients," Journal of the American Society of Echocardiography, vol. 27, no. 10, pp. 1113-1119, 2014.

[53] L. Cortigiani, F. Rigo, S. Gherardi et al., "Coronary flow reserve during dipyridamole stress echocardiography predicts mortality," JACC: Cardiovascular Imaging, vol. 5, no. 11, pp. 1079-1085, 2012.

[54] V. L. Murthy, M. Naya, C. R. Foster et al., "Improved cardiac risk assessment with noninvasive measures of coronary flow reserve," Circulation, vol. 124, no. 20, pp. 2215-2224, 2011.

[55] V. Kunadian, A. Chieffo, P. G. Camici et al., "An EAPCI expert consensus document on ischaemia with non-obstructive coronary arteries in collaboration with European society of cardiology working group on coronary pathophysiology \& microcirculation endorsed by coronary vasomotor disorders international study group," European Heart Journal, vol. 41, no. 37, pp. 3504-3520, 2020.

[56] D. F. Bechsgaard, I. Gustafsson, M. M. Michelsen et al., "Vital exhaustion in women with chest pain and no obstructive coronary artery disease: the iPOWER study," Evidence Based Mental Health, 2020. 
[57] L. Jespersen, S. Z. Abildstrom, A. Hvelplund et al., "Symptoms of angina pectoris increase the probability of disability pension and premature exit from the workforce even in the absence of obstructive coronary artery disease," European Heart Journal, vol. 34, no. 42, pp. 3294-3303, 2013.

[58] T. J. Ford, B. Stanley, R. Good et al., "Stratified medical therapy using invasive coronary function testing in angina," Journal of the American College of Cardiology, vol. 72, no. 23, pp. 2841-2855, 2018.

[59] F. Crea, P. G. Camici, and C. N. Bairey Merz, "Coronary microvascular dysfunction: an update," European Heart Journal, vol. 35, no. 17, pp. 1101-1111, 2014. 